\title{
Alcohol Hangover- its Effects on Human Body: Review
}

\author{
Loveleen Bajaj* and Ranjeet Singh \\ Department of Food science \& technology, Khalsa College, India \\ Received: May 20 2018; Published: May 25, 2018 \\ *Corresponding author: Loveleen Bajaj, Department of Food science \& technology, Khalsa College, Amritsar, India
}

\begin{abstract}
A hangover is a syndrome of physical and mental symptoms that occurs after 8 to 16 hours of consumption with a zero level of alcohol. The objective of the study is to explore effects of the alcohol hangover. The impairing effects on memory functioning such as delayed recall, irritation, lack of concentration and after acute alcohol intoxication. Normally the body might use cytokines to trigger fever of inflammatory response to battle infection leading to unpleasant effects include nausea, vomiting, dizziness, fatigue and hormonal imbalance in the body. Dehydration is believed to be the cause of hangover as it leads to anti-diuresis state in the body. The most compelling theory that, at the moment, is that hangover results from a buildup of acetaldehyde, a toxic compound in the body. The congener amount in the particular alcoholic beverage is believed to be increase the extent of severity of hangover. Methanol, found in highest levels in whiskey and red wine has received a larger amount of blame for showing that it can linger in the body after all alcohol has been eliminated, perhaps accounting for the enduring effects of hangover.
\end{abstract}

Abbreviations: BAC: Blood Alcohol Concentration; AW: Alcohol Withdrawal; FASD: Fetal Alcohol Spectrum Disorders

\section{Introduction}

Alcohol hangover is defined as the changes that occur after the consumption and full metabolism of alcohol with sufficient severity to disrupt the performance of daily tasks and responsibilities. It is characterized by headache, tremulousness, nausea, diarrhea and fatigue combined with decreased occupational, cognitive or visual spatial performance. Moreover the hangover after effects are dependent on the type of alcoholic beverage taken and the quantity the person drinks. Typically, hangover starts within few hours after the winding up of drink consumed when the level of blood alcohol concentration (BAC) is declining. The severity of the effect of hangover usually increase when the BAC is zero and 24 hours thereafter. Generally, the hangover is more pervasive when the both the duration and amount of beverage consumed is greater. A survey of Hardburg and his fellow workers in 1993 the pervasiveness of hangover was found out that nearly $75 \%$ of the persons who consumed till drunkenness developed hangover at least few times. In the second survey, the co relation between the alcohol intake per week and the intensity of the hangover as founded by 160 Finnish men $.43 .8 \%$ of group of harvest drinkers (who consumed greater than 106g of alcohol every week or 9drinks) when investigated were found undergoing a hangover over a month or more in comparison to the persisting other persons taken under study as stated by Kauhanen in1997 other study surveys found out that in case of heavy drinkers less hangover is experienced.
Anes and colleagues revealed that half of the interrogated workers during hangover were at the work. Throughout the hangover period, the workers were not only sick but also had disputes with their co -workers and their supervisors, slumbered at work and find difficulty in completing the work. Decreased proficiency is normal when hangover takes place at work. Studies confirmed that beverage with higher congener content produce more severe hangover symptoms. In the beginning of the alcohol intake the sleep encountering period is delayed, and can extend the total sleep time at reduced doses of $0.16 \mathrm{~g} / \mathrm{kg}$ unlike the limited and increased doses Although ethanol is the main element of hangover problem (eg. Chapman, 1970; Ylikahri e. al. in 1974) the severity symptoms may be increased by congeners. Laurell and Tornos (1983) obtained a $20 \%$ decrease in psycomotor ability in drive task at 3 hours after $\mathrm{BAC}$ reached 0 when a high dose was administered. Anderson and Dawson in 1999 demonstrated that the driving ability was altered during the alcohol hangover, illustrated by the failure of the collection of the psychical resources on the completing stimuli. Petros et.al.in 2003 illustrated the likeliness that hangover can induce effects only in some capabilities required for flight performance. The affirmation of mild withdrawal is due to the lapping over between mild alcohol withdrawal (AW) symptoms and hangover. Even a single bout of drinking hangover can take place, in contrast after withdrawal the risk of injury and task performance is affected. 


\section{Methodology}

When the blood alcohol concentration (BAC) returns to zero the alcohol hangover is developed and it is indicated by a feeling of discomfort that last for more than 24hours. Several features are thought to be included like those of alterations in immune system, and metabolism of glucose, dehydration as well as the acetaldehyde accumulation. Hangover is suggested to be early stage of alcohol withdrawal. Acetaldehyde, a breakdown product of alcohol metabolism, plays a role in producing symptoms. Chemicals formed during alcohol processing and maturation known as congeners increase the frequency and severity of hangover. In the fermentation process along with the ethanol the congeners are produced, formed during the decomposition of the organic components of the beverage or included to the beverage in the production process . They contribute to taste, smell and also contribute to the flavor of the alcoholic beverage. Liquors such as brandy, wine, tequila, whiskey and other dark liquors containing congeners tend to produce severe hangover. Whereas the clear liquors such as rum, vodka, gin cause hangover less frequently. Alcohol dehydrogenase produces acetaldehyde which is toxic which is converted to acetic acid which is later converted to fatty acid and water. Also, alcohol dehydrogenase produces methanol (hangover causing congener) gets converted to formaldehyde and formic acid.

The chronically administered depressant substances cause the changes or alterations in the central nervous system. Hence the alterations included in such kind of changes are the embedded two types of receptors in the nerve cell membranes. One type receptors that attach with the necessary chemical messenger that is the neurotransmitter called the gamma aminobutyric acid or GABA and distinct kind binds with other neurotransmitter i.e glutamate. The nerve cell activity is critically regulated by both glutamate and GABA .With the increasing exposure of the body to the chronic alcohol the count and sensitivity of glutamate receptors decline in an attempt to neutralize sedative effect of alcohol. The portion of the central nervous system and nervous system that harmonize responses to stress remain in unbalanced "overdrive" condition when the alcohol is removed from the body as said by Tsai in 1995 .Thereby the excitability of the sympathetic nervous system that cause tremors, sweating, increase in heart rate than the normal resting rate is remarked in both hangover and alcohol withdrawal syndrome. After being injested, the enzyme alcohol dehydrogenase converts acetaldehyde to ethanol and then by egestion and oxidation into acetic acid. This reaction also converts with the help of redox reaction as the NADH is being reduced from NAD+. The alcoholic beverage makes the bodily functions more difficult by causing an imbalance in the NAD+ and NADH redox system.

\section{Effects on human body}

Urine production gets accelerated due to the dehydrating effect of ethanol i.e dieresis which can further cause dry mouth, dizziness, thirst and may result in electrolyte imbalance .The genesis of alcohol hangover is indicated by studies and are originated by the dehydration effects. In the pathogenesis of hangover hyperglycemia has been assumed to play a crucial role. It is because of the hinderance of vasopressin released from posterior pituitary gland. During withdrawal from alcohol vasopressin is released resulting in water retention (antidiuresis). Few researches suggest that the reason behind memory impairment is dehydration (eg. Cian etal.2001; Tomporowski, 2003; Lieberman etal.2005) There is a vital co- relation between immune factor and hangover is the most compelling factor studied. Excessive alcohol consumption makes the immune system feeble making body confront a disease with more ease. The variation in the immune system in regard of cytokine metabolism has been recognized as playing a vital role in pathophysiology of the state of hangover. Mainly the symptoms of the hangover nausea, headache and fatigue have been put forward to be arbitrated by variations in the immune system .After the consumption of alcohol the level of several cytokines have been found to significantly incline in the blood. It includes interleukin 12, interferon gamma and interleukin. Intake of the intoxication can lower the rate of body's ability to generate cytokines that avert infections by inducing inflammations. The capacity of white blood cells to effectively engulf and consume harmful bacteria is diminished by the use of chronic alcohol. Alcohol jumbles the process of directing the enzymes for metabolism of food to the small intestine. It results in the secretion of digestive juices by the pancreas internally instead of sending enzymes to small intestine.

These enzymes along with the acetaldehyde - a substance generated by metabolizing or breaking down to alcohol -are detrimental to the pancreas. Alcohol causes inflammation of the stomach lining and hindering the process of emptying the stomach content which actually irritates the stomach and intestine. Along with increase in the level of pancreatic and intestinal secretions it also results in making of more gastric acid Upper abdominal pain, nausea, vomiting experienced during hangover can be caused by any of these elements. Alcohol effects metabolic processes involving insulin and also harms the pancreatic cells. The dangerous inflammations are kept open by this process in the pancreas. In the absorption of alcohol, acetaldehyde dehydrogenase is produced which being a chemically reactive matter attach not only with the proteins but also other biologically essential compounds. At greater intensity, toxic effects are caused like those of nausea, vomits, skin reddening and pulse rate. In order to prevent the accumulation of intermediate metabolite in higher concentration in most people ALDH metabolizes the acetaldehyde effectively and swiftly, whereas limited amounts are existent in blood during drunkeness.

The aggregation of the acetaldehyde granted by the ALDH enzyme in few people is due to genetic variations .Such individuals on the routine basis experience sweating, flush and become sick after the intake of little amounts of alcohol. The tranquillizing effect of the alcohol induces onset of sleep and the disturbing effect of alcohol on sleep during the hangover state results in fatigue. Walsh in 1991 stated that rebound excitation causes the sleep to be experienced after the induction of the alcohol to be of shorter length of time and poor quality. Alcohol de-stress the muscles of the throat that results in intensification of snoring activity and likely the periodic interruption of the breathing i.e. sleep apnea. The higher the intake of alcohol, the shorter the corresponding sleep latency. Polysomnograpic assessments revealed that the consumption of alcohol leads to reduction in sleep proficiency and rapid movement 
of eyes during sleep, and intensification of the weak duration and latterly self -reported sleepiness. Hangover severity was worse in subjects with higher hangover severity scores had a decline in their sleep time and devoted lesser time in the rapid movement of eye during sleep. In the hangover period, the continuation of the intoxicant discommodes with the various other biological rhythms too. For an illustration ,the normal 24hours rhythms in the body temperature is disorganized by alcohol, comprising a body temperature which is unusually low at the time of drunkenness and unusually high at the time of withdrawal.

The circadian nighttime secretion of the growth hormone is obstructed by the alcohol intoxication which is otherwise essential for the synthesis of proteins and bone growth. In contradiction, alcohol influence the liberation of the hormone from the pituitary gland i.e. adrenocorticotrophic which successively encourage the liberation of cortisol , a hormone that helps in the regulation of the orderly circadian increase and decline of the cortisol level .As stated by Gauvin in 1997 in general the alcohol's disorganization of the circadian rhythms promote 'jet lag' and the hypothesis has been put forward that this is the reason behind some of the adverse effects of the alcohol. The rate of effectiveness of functions of the brain declines during hangover accompanied by dizziness, nausea and anxiety. The rate of conveying information that links the neurotransmitters in the brain which are important for translation of information to the rest of body is declined by the intake of alcohol. The certain representative symptoms of withdrawal like the failure to concentrate, decrease in appetite and lack of interest in normal day to day tasks are the result of alcohol's stimulation of inflammatory response to the immune system .It is found that increase of $30 \%$ of the performance error among hangover participants and overall 5-10\% drop in working memory. The memory retrieval processes are significantly impaired during alcohol hangover. There have been proclamations frequently about the memory and psychomotor wreckage during the intoxication of alcohol (Ferrara in 1994 ;Kruger, 1992; Holloway, 1995; Koelega, 1995; Moskowitz and Fiorentino, 2000). The impairing effects on memory retrieval processes are in line with those observed after observed after acute alcohol intoxication and those found in alcoholics. The sympathetic nervous system is disorganized greatly by the alcohol which otherwise along with other things is responsible for controlling the constriction and dilation of the vessels of blood for countering anxiety, temperature as well as exertion, etc .Particularly the ample intake of alcohol and unhealthy disturbance in eating behavior leads to acceleration of blood pressure. The consequence of such a thing over time can eventually be chronic. Health problems such as renal diseases, heart diseases and stroke are the result of the high blood pressure. Alcohol containing drinks can decrease the blood pressure of the body by widening of the blood vessels that supply to the brain. These changes can produce pain and sensitivity, resulting in headache .A negative mood before drinking alcohol can predispose some people to migraine headaches independent of what they drink .A headache may begin within few hours after drinking or starts after 16 hours later.

Drinking seems to increase both systolic and diastolic blood pressure during intoxication but not during hangover. During the period when blood alcohol levels are decreasing, usually at night, both pressure levels fall to less than the basic level. Out of all the organs that are damaged by heavy alcohol use, the liver often suffers the most, typically through alcoholic hepatitis which involves swelling ,pain and cirrhosis (irreversible scarring of liver)effects between $15-30 \%$ of the people who abuse alcohol. The brain as well as the liver function both are affected by the alcoholic liver disease .The majority of alcohol consumed by the person is broken down by the liver. The formation of the toxins during the breaking process of alcohol is more dangerous than alcohol itself the liver cells are damaged by the byproducts of alcohol by this process. The toxic substances, ammonia and manganese particularly move to the brain and this is allowed by the damaged liver cells that no longer function .Hepatic encephalopathy -a deadly and critical brain disorder in which damage of brain cells occurs is the result of these substances .Ultimately, these conditions can disorganize the metabolism of the body and spoil the functions of other organs too. Owing to liver's essential role in detoxification of alcohol it is specifically accessible to harm from too much alcohol. Alcoholism can cause a thiamine (vitamin B12) deficiency which can cause rapid eye movements, weakness or paralysis to eye muscles. Consuming larger quantities of alcohol on a regular basis can interfere with the amount of thiamine absorbed by the body by reducing thiamine uptake in the gut, and by preventing cells from absorbing adequate amounts of vitamin. Alcohol has huge effect on the fetal development. At any stage i.e. even before birth the brain can be affected. It occurs due to prenatal alcohol exposure. A range of problems called fetal alcohol spectrum disorders (FASD) can occur.

FASD symptoms include baby's physical abnormalities, learning difficulties and emotional problems, can often last lifetime. The overall growth and brain function is decreased. For women, the risk of breast cancer rises with alcohol use. Alterations of the somatic growth and specific minor inflammations are most characteristic. The central nervous system is greatly affected. It makes harder to talk, causing slurred speech. It also affects coordination, interfering with balance and ability to walk. In longer term, drinking can actually shrink the frontal lobes of the brain. Acute alcohol withdrawal can lead to seizures and delirium. And severe alcoholism can progress to permanent damage, causing dementia. Damage to nervous system can result in pain, numbness or abnormal sensations in feet and hands. A nerve impairment known as alcoholic neuropathy in which hurting pins and needle type of sensation or paralysis like in extreme cases as well as in case of weak muscles is experienced and in abstinence erectile dysfunction and constipation can take place. The origination of alcoholic neuropathy is due to the toxicity of alcohol to nerve cells or on account of nutritional scantiness owing to ample intake of alcohol compromising nerve function .It can affect both movement and sensation. Symptoms range from slight discomfort to major disability. Areas of the body affected by alcoholic neuropathy are arms and legs, urinary and bowel, impotency, heart intolerance, vomiting, nausea, dizziness and impaired speech. Alcohol can cause blood sugar level to fall. If the blood sugar level declines tiredness, feebleness, disturbance of mood and in some cases even convulsions are experienced. 


\section{Conclusion}

Hangover, a common disorder, has substantial morbidity and societal cost. Recent studies reveal that the alcohol hangover induces the disturbance in the electrolyte balance ultimately leading to dehydration. The unevenness particularly the cytokine metabolism in the immune system plays a vital role. The biological rhythms are also affected leading to disturbance in the sleep pattern. Alcohol actually slow down the pace of communication resulting in slurred speech, nausea, headache, etc. Hallucinations are experienced by one fourth of the alcohol dependents on withdrawal. Due to extreme reactivity, alcohol has the potential to affect virtually every organ or biochemical pathway in the human body. These adverse changes arise due to the extreme biochemical or chemical activity of ethanol itself or its reactive metabolite acetaldehyde. In addition to the biological effects of ethanol the psychological and socio-economical effects are disastrous for patients in chronic alcoholism. The aim is to change the negative effect of congeners and other by products in such a way to stop the harmful effect on body.
This work is licensed under Creative Commons Attribution 4.0 License

Submission Link: https://biomedres.us/submit-manuscript.php

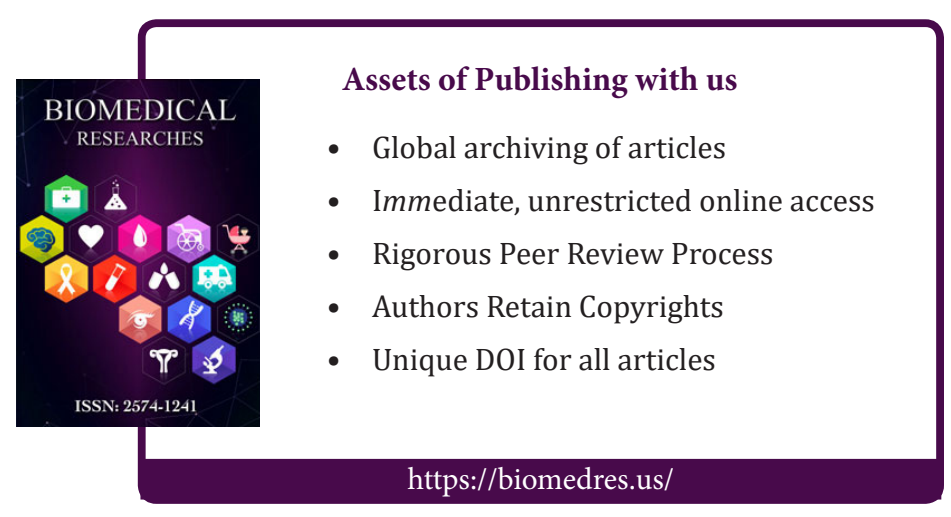

